\title{
Universal probe-based intermediate primer- triggered qPCR (UPIP-qPCR) for SNP genotyping
}

\author{
Baowei $\mathrm{Li}^{1,2^{*}} \mathbb{D}$, Yanran Liu ${ }^{3}$, Xiaodan Hao ${ }^{2}$, Jinhua Dong ${ }^{1}$, Limei Chen ${ }^{1}$, Haimei Li ${ }^{1}$, Wei $\mathrm{Wu}^{4}$, Ying Liu²,
} Jianxun Wang ${ }^{3}$, Yin Wang ${ }^{2}$ and Peifeng $\mathrm{Li}^{2^{*}}$

\begin{abstract}
Background: The detection and identification of single nucleotide polymorphism (SNP) is essential for determining patient disease susceptibility and the delivery of medicines targeted to the individual. At present, SNP genotyping technology includes Sanger sequencing, TaqMan-probe quantitative polymerase chain reaction (qPCR), amplification-refractory mutation system (ARMS)-PCR, and Kompetitive Allele-Specific PCR (KASP). However, these technologies have some disadvantages: the high cost of development and detection, long and time consuming protocols, and high false positive rates. Focusing on these limitations, we proposed a new SNP detection method named universal probe-based intermediate primer-triggered qPCR (UPIP-qPCR). In this method, only two types of fluorescence-labeled probes were used for SNP genotyping, thus greatly reducing the cost of development and detection for SNP genotyping.
\end{abstract}

Results: In the amplification process of UPIP-qPCR, unlabeled intermediate primers with template-specific recognition functions could trigger probe hydrolysis and specific signal release. UPIP-qPCR can be used successfully and widely for SNP genotyping. The sensitivity of UPIP-qPCR in SNP genotyping was $0.01 \mathrm{ng}$, the call rate was more than $99.1 \%$, and the accuracy was more than $99.9 \%$. High-throughput DNA microarrays based on intermediate primers can be used for SNP genotyping.

Conclusion: This novel approach is both cost effective and highly accurate; it is a reliable SNP genotyping method that would serve the needs of the clinician in the provision of targeted medicine.

Keywords: UPIP-qPCR, Intermediate primer, Universal probe, SNP genotyping, Sanger sequencing

\section{Background}

In a clinical setting, single nucleotide polymorphism (SNP) genotyping provides a door into recognizing individual susceptibility to disease and underpins the prospect of targeted medicine [1-4]. Currently, SNP

\footnotetext{
*Correspondence: Ibwwbl@126.com; peifli@qdu.edu.cn

'Key Laboratory of Biological Medicines in Universities of Shandong Province, Weifang Key Laboratory of Antibody Medicines, School of Bioscience and Technology, Weifang Medical University, Jinan 261053, Shandong, China ${ }^{2}$ Institute for Translational Medicine, Qingdao University, Qingdao 266021, China

Full list of author information is available at the end of the article
}

genotyping technology mainly includes first-generation sequencing, TaqMan probe quantitative polymerase chain reaction (qPCR), amplification-refractory mutation system (ARMS)-PCR, and Kompetitive Allele-Specific PCR (KASP [5-7]. However, these technologies have limitations such as high cost of development and detection, long and time consuming protocols, and high false positive rates $[5,8]$.

Sanger sequencing is very accurate and could be considered the gold standard for the identification and detection of SNPs $[9,10]$. However, Sanger sequencing

C C The Author(s). 2021 Open Access This article is licensed under a Creative Commons Attribution 4.0 International License, which permits use, sharing, adaptation, distribution and reproduction in any medium or format, as long as you give appropriate credit to the original author(s) and the source, provide a link to the Creative Commons licence, and indicate if changes were made. The images or other third party material in this article are included in the article's Creative Commons licence, unless indicated otherwise in a credit line to the material. If material is not included in the article's Creative Commons licence and your intended use is not permitted by statutory regulation or exceeds the permitted use, you will need to obtain permission directly from the copyright holder. To view a copy of this licence, visit http://creativecommons.org/licenses/by/4.0/ The Creative Commons Public Domain Dedication waiver (http://creativecommons.org/publicdomain/zero/1.0/) applies to the data made available in this article, unless otherwise stated in a credit line to the data. 
requires expensive equipment, running costs (for example reagent costs) are high, and workflows are laborious and slow; consequently, Sanger sequencing is not well suited to a clinical setting. TaqMan probe-based qPCR, the most widely used SNP detection method in clinical diagnostics, is rapid, highly accurate and has a low cost per assay. However, probe optimization is a complex and slow process, making marker development expensive. This has limited its application in large-scale SNP genotyping [11-15].

ARMS-PCR is an amplification-refractory mutation system $[16,17]$. ARMS-PCR has a tendency to produce false positives when the number of cycles of amplification is high, thus significantly limiting the application of this method in clinical detections [18].

Universal template probe assays based on qPCR [19] and KASP [20], unlike TaqMan, lack specific-sequence primers and usually result in false-positive results, which makes them difficult to use for clinical examinations [21]. SNP arrays are able to detect large numbers of SNPs in a single assay but they are expensive and protocols are long and laborious, which making it unsuitable for clinical individualized SNP genotyping [5, 22].

In view of the limitations of the above technologies and the actual needs of clinical SNP detection, we established a new and improved SNP detection method, named universal probe-based intermediate primertriggered qPCR (UPIP-qPCR). In the development of any type of SNP genotyping kit based on this method, two types of universal fluorescence probes are used, and intermediate primers are introduced to guarantee specificity, so that the cost and duration of research and development for genotyping kits are significantly reduced, and UPIP-qPCR gives rise to the need for low cost and high accuracy of clinical SNP genotyping.

\section{Results}

Feasibility verification of UPIP-qPCR in SNP genotyping

Universal probe-based and intermediate primertriggered qPCR (UPIP-qPCR) is a two-step process. The first step, a standard PCR reaction, takes c. 30 mins. The second step, the qPCR reaction which uses the product of the first step, takes c. 60 mins, to obtain the corresponding fluorescence signals of the alleles (Fig. 1a and Fig. S1a).

We used rs671 and rs2031920 loci as candidate SNPs to verify the feasibility of UPIP-qPCR and optimize this technology. Following the reaction, the corresponding genotypes were identified according to the final fluorescence category and intensity (relative fluorescence units, RFU). The results showed a typical S-type amplification curve with exponential growth and the amplification signals of the three positive DNA standards were specific. The amplification signals of the GG genotype were only
FAM-positive, the GA genotype was both FAM- and HEX- positive, the AA genotype was only HEX-positive, and the no-template control group (NTC) had no falsepositive signals, indicating the accuracy of the genotyping results (Fig. 1b and Fig. S1b). These results indicate that UPIP-qPCR is feasible for SNPs genotyping. As a new genotyping method, its time and cost consumption are less than those of TaqMan probe-qPCR and Sanger sequencing, and its operation complexity is moderate (Table 1). Thermal cycles, primer concentrations and products dilution ratios of the first stage play important roles in the UPIP-qPCR, and the PCR products should be diluted 10 times before used in the second stage (Fig. S2).

\section{UPIP-qPCR presented high sensitivity in SNP genotyping detections}

The sensitivity of UPIP-qPCR was analyzed using a primer concentration of $100 \mathrm{nM} /$ each and 18 cycles of amplification in the first-stage reaction; human genomic DNA samples of three genotypes of rs671 with different concentrations were used. The results showed that most of the DNA samples with different concentrations presented typical S-type curves, and the order of appearance of the curves of amplification (S-shaped curves) was inversely related to the concentration of DNA in the samples (Fig. 2a, c and e). The accuracies of all the concentrations of three genotypic genomic DNA were $100 \%$, and although there were good call rates in the high concentration samples, it was not ideal in the low concentration samples (Fig. 2b, d and f). Specifically, the call rates of all three genotypes were $100 \%$ at five concentration gradients from $100 \mathrm{ng} / 10 \mu \mathrm{L}$ to $0.01 \mathrm{ng} / 10 \mu \mathrm{L}$, and the copy number gradients from 33,000 genomic DNA per $10 \mu \mathrm{L}$ to 3 genomic DNA per $10 \mu \mathrm{L}$. The other call rates were: $100 \%$ for GG and AA samples with a concentration of $0.003 \mathrm{ng} / 10 \mu \mathrm{L}, 72.22 \%$ for GA samples with a concentration of $0.003 \mathrm{ng} / 10 \mu \mathrm{L}, 27.78,11.11$ and $22.22 \%$ for GG, GA and AA samples with a concentration of $0.001 \mathrm{ng} / 10 \mu \mathrm{L}$, respectively (Fig. $2 \mathrm{~b}, \mathrm{~d}$ and f). The above data showed that the concentration of 0.01 $\mathrm{ng} / 10 \mu \mathrm{L}$ has the highest sensitivity of UPIP-qPCR, i.e., every $10 \mu \mathrm{L}$ reaction system containing three copies of genomic DNA can obtain reliable genotyping results.

TaqMan probe-qPCR was performed to compare the sensitivity of the two methods. The results showed that DNA samples with high concentrations presented typical S-type curves, (Fig. S4a, S4c and S4e). The accuracies in three genotypic genomic DNA with concentrations from $100 \mathrm{ng} / 10 \mu \mathrm{L}$ to $0.01 \mathrm{ng} / 10 \mu \mathrm{L}$ were $100 \%$ (Fig. S4b, S4d and S4f). Call rates in three genotypic genomic DNA with concentrations from $100 \mathrm{ng} / 10 \mu \mathrm{L}$ to $0.1 \mathrm{ng} / 10 \mu \mathrm{L}$ were $100 \%$, but were $<100 \%$ or even $0 \%$ in concentrations from $0.03 \mathrm{ng} / 10 \mu \mathrm{L}$ to $0.01 \mathrm{ng} / 10 \mu \mathrm{L}$ (Fig. S4b, S4d 
$\mathrm{a}$
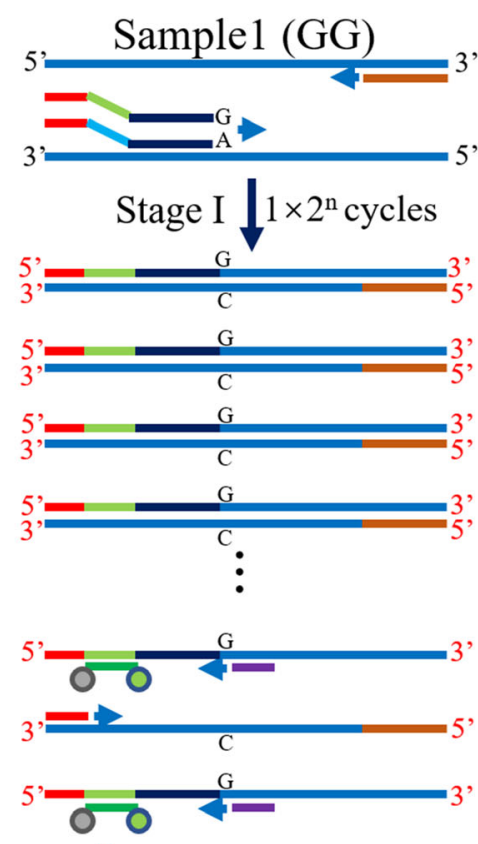

3 ,
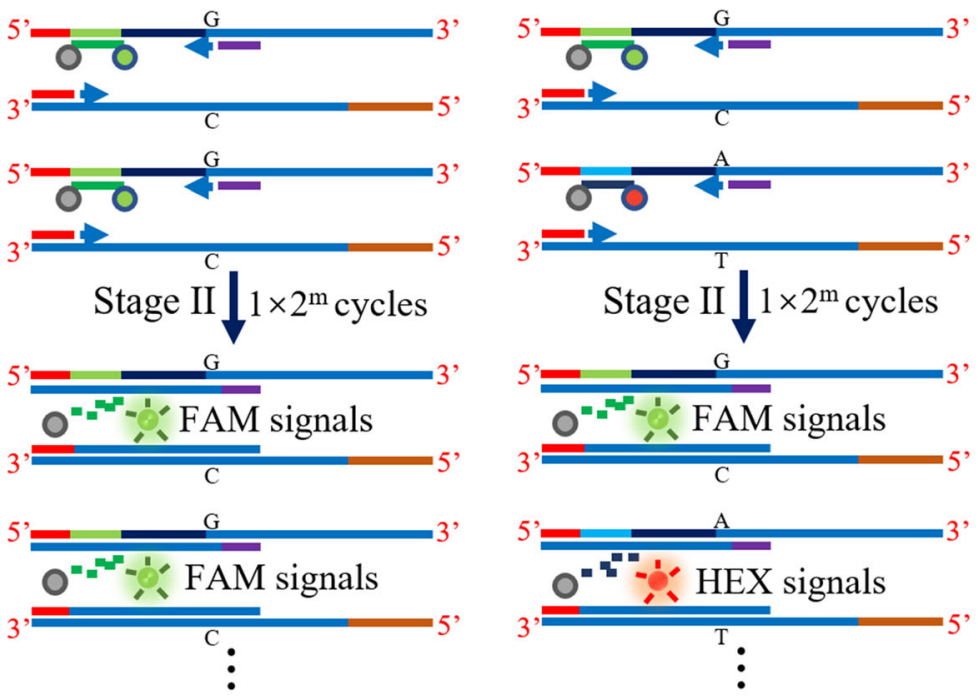

Stage II $\downarrow^{\mathrm{T}} 1 \times 2^{\mathrm{m}}$ cycles
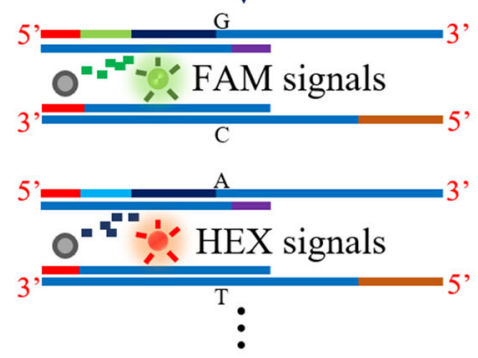

Universal primer

Upstream specific primer sequence

Downstream specific primer

Q OQ Universal probes (UP)

L Reverse complementary sequence of UP

b
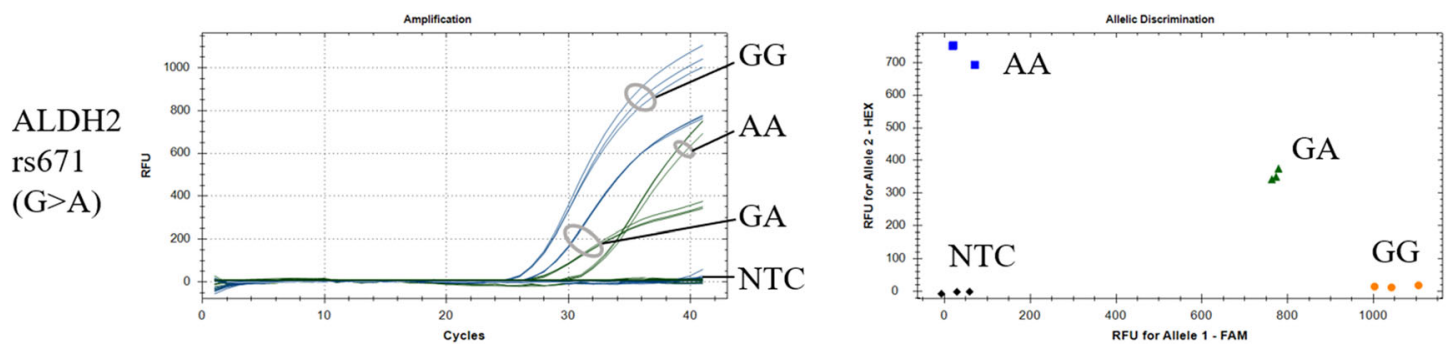

Fig. 1 (See legend on next page.)
- Intermediate primer
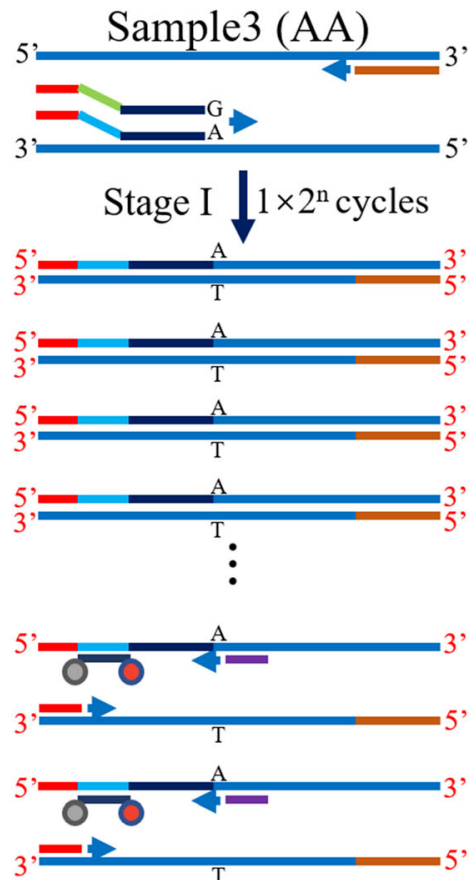

Stage II $\downarrow 1 \times 2^{\mathrm{m}}$ cycles
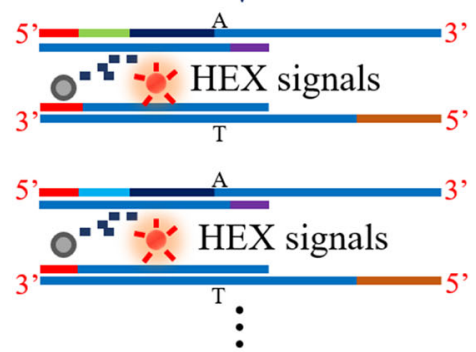

$\rightarrow$ Direction of elongation

ㅍniversal connectors

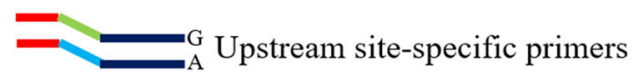
$\mathrm{n}=10 \sim 22 ; \mathrm{m}=35 \sim 40$ 
(See figure on previous page.)

Fig. 1 Principle and application of UPIP-qPCR for SNP genotyping. The reactions of UPIP-qPCR are divided into two stages (a). The first stage is a general PCR reaction aiming to obtain a certain amount of DNA fragments containing specific SNP sites (a, upper panel in each sample). The second stage is a quantitative PCR (qPCR) reaction, which uses the PCR products of the first stage as templates to obtain the corresponding fluorescence signals of alleles (a, lower panel in each sample). UPIP-qPCR was initially used to identifying the SNP genotypes of ALDH2 rs671 (b). All three genotypes of each SNP samples successfully yielded accurate results by UPIP-qPCR. The homozygous WT genotypes presented by FAMonly signals (blue curves) were shown in the lower right area of the genotyping scatter diagrams with yellow dots, the heterozygous genotypes presented by FAM and HEX signals (blue curves and green curves) were shown in the middle area of the genotyping scatter diagrams with green triangles, and the homozygous mutant genotypes presented by HEX-only signals (green curves) were shown in the upper left area of the genotyping scatter diagrams with blue squares. No-template control groups (NTC) were shown in the lower left area of the genotyping scatter diagrams with black rhombuses. Each sample was detected with three duplicates in one experiment, and the experiments were repeated more than three times

and S4f). These data showed that the concentration of $0.1 \mathrm{ng} / 10 \mu \mathrm{L}$ was the highest sensitivity of TaqMan probe-qPCR, i.e., every $10 \mu \mathrm{L}$ reaction system containing 33 copies of genomic DNA can obtain reliable genotyping results by TaqMan probe-qPCR method.

\section{UPIP-qPCR possessed high call rate and accuracy}

In this study, the genotypes of rs671, rs1057910, rs9923231, rs1801131, rs1801133, and rs1801394 in 224 DNA samples were detected by UPIP-qPCR, TaqMan probe-qPCR, KASP and Sanger sequencing. The UPIPqPCR scatter plots showed good signal differentiation. Each scatter diagram contained three repeats of positive standards and NTC. The yellow dots represent the wildtype genotype, the green triangles represent the heterozygous genotype, and the blue squares represent the mutant genotype (Fig. S5a). The TaqMan probe-qPCR scatter plots also showed good signal differentiation in the genotyping detection of these six SNPs (Fig. S5b). The KASP scatter plots showed many undefined dots (Fig. S5c). The call rates of rs671, rs1057910, rs9923231, rs1801131, rs1801133, and rs1801394 generated by UPIP-qPCR were 99.11, 100, 100, 100, 99.55 and $100 \%$ respectively, which were all higher than those of TaqMan probe-qPCR and KASP (Table 2). Compared with Sanger sequencing results (Additional file 3), the accuracies of UPIP-qPCR and TaqMan probe-qPCR were all $100 \%$ in the detection of these six SNPs, and the accuracies of KASP were all below $100 \%$ (Table 2). By counting the allele frequency, the minor allele frequency (MAF) of 224 samples detected by UPIP-qPCR was similar to MAF values of East Asians derived from ALFA or 1000 Genomes (NCBI) (Table S7), which indicated that UPIP-qPCR has sufficient ability to recognize the distribution of SNP genotypes in a specific human population.

\section{UPIP-qPCR recognizes all SNP variants and InDels}

There are six types of nucleotide alterations in point mutations, including interchanges between A-G, A-C, A-T, G-C, G-T and C-T. InDels are defined as the insertion or a deletion of one or more nucleotides into a DNA sequence with respect to a defined reference sequence. In addition to the A-G, A-C and C-T mutation types involved in the above experiments, we also selected other SNPs of all mutation types to test the wide adaptability of UPIP-qPCR. The genes and SNP sites were as follows: $A B C B 1$ rs10234411 (A > T), ADD1 rs4961 (G > T), ADRB1 rs1801253 (G > C), MTHFR rs1801131 (A > C), MTHFR rs1801133 $(\mathrm{C}>\mathrm{T}), \quad M T R R \quad \mathrm{rs} 1801394(\mathrm{~A}>\mathrm{G}), A B C B 1$ rs1045642 $(\mathrm{T}>\mathrm{C}), \quad D P Y D \quad \mathrm{rs} 3918290 \quad(\mathrm{G}>\mathrm{A}), \quad D P Y D$ rs55886062 (A > C), GSTP1 rs1695 (A > G), XRCC1 rs25487 (A > G), APC rs35305379 (TTTA > TTTTA), APC rs34481414 (ACTACAAT > ACAAT). These SNPs are of significance in determining individual responses to medical treatments. The results showed that UPIP-qPCR was able to identify all SNP types regardless of whether they were transitions of transversions (Fig. 3a and Fig. S6a). The clustering of fluorescent signals for the homozygous and heterozygous calls was distinct and clear in the scatter diagrams (Fig. 3b and Fig. S6b), and the results were accurate. These results suggests that UPIP-qPCR can be widely used to genotype different SNPs and InDels.

\section{Microarrays based on intermediate primers were feasible for SNP genotyping}

As the intermediate primers were able to recognize the template DNA specifically, $20 \times 5$ dot DNA microarrays

Table 1 Characteristics of Three Methods for SNP Genotyping

\begin{tabular}{llll}
\hline & UPIP-qPCR & TaqMan & Sanger \\
\hline Reagent cost for kit development (\$/SNP) & $35-70$ & $500-1000$ & $10-20$ \\
Reagent cost for detection (\$/SNP) & $\sim 0.3$ & $\sim 1$ & $\sim 3$ \\
Detection period (hours/SNP) & $1.5-2$ & $1.5-2$ & $12-24$ \\
Operation complexity & Moderate (Two stages) & Low (One stages) & High (Five stages) \\
\hline
\end{tabular}



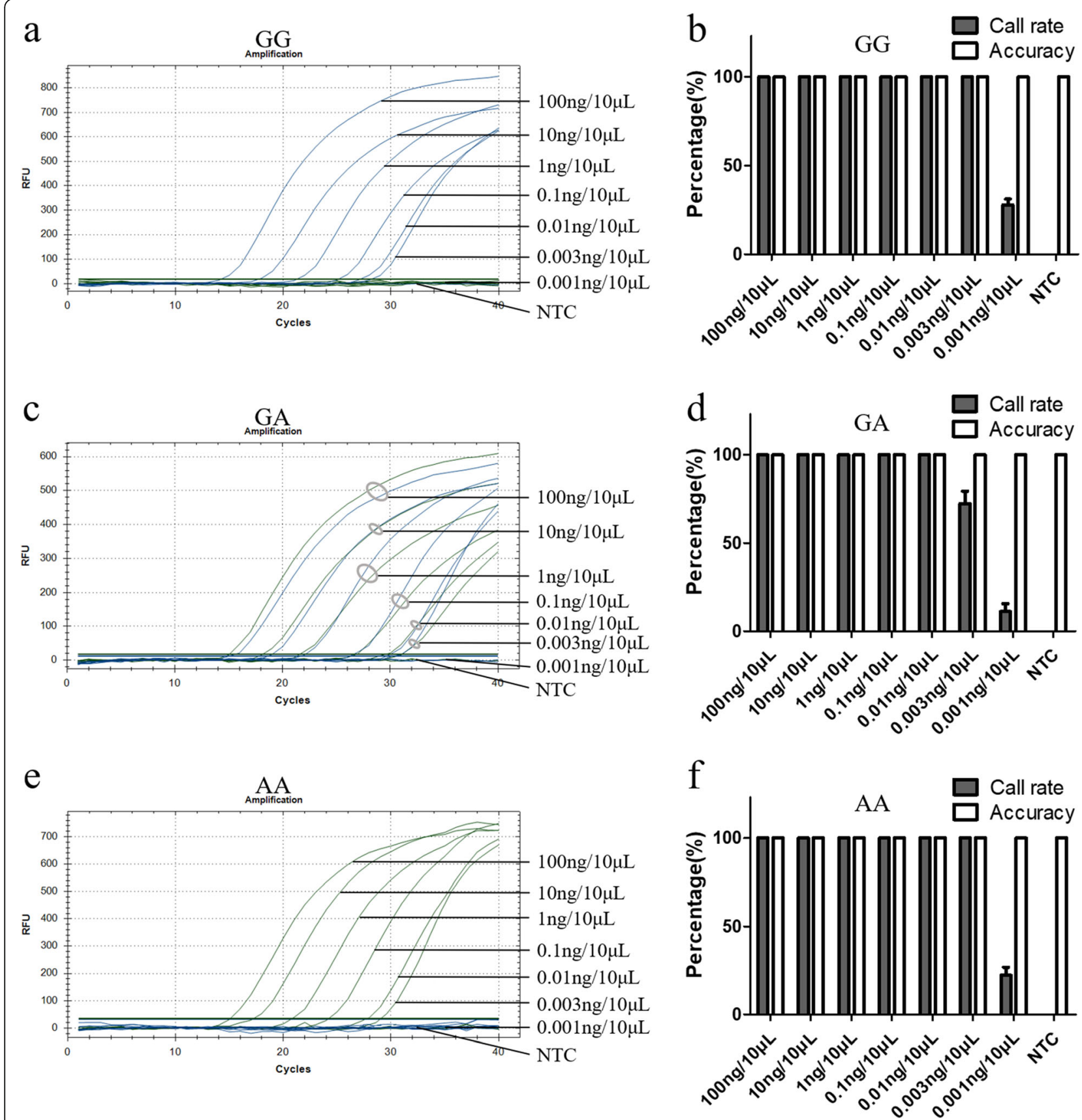

Fig. 2 Sensitivity analysis of UPIP-qPCR. Three genotypic DNA samples of ALDH2 rs671 with concentrations from $100 \mathrm{ng} / 10 \mu \mathrm{L}$ to $0.003 \mathrm{ng} / 10 \mu \mathrm{L}$ showed typical S-type curves, and the appearance order of the curves was positively correlated with the decrease in concentration gradient (a, $\mathrm{C}$ and e). The call rates were $100 \%$ in all three genotypic samples with concentrations from $100 \mathrm{ng} / 10 \mu \mathrm{L}$ to $0.01 \mathrm{ng} / 10 \mu \mathrm{L}$, but $<100 \%$ in concentrations of $0.003 \mathrm{ng} / 10 \mu \mathrm{Land} 0.001 \mathrm{ng} / 10 \mu \mathrm{L}(\mathrm{b}, \mathrm{d}$ and f). The accuracy of all three genotypic genomic DNA concentrations was $100 \%$ (b, $d$ and f). FAM and HEX signal curves of the same concentration in genotype GA were indicated by a gray ellipse. Each sample was detected with nine duplicates in one reaction, and the experiments were repeated four times. Bars show SD $(n=36)$

were made (Fig. 4a) with 16 types of SNP intermediate primers, positive primers, negative primers, and internal reference (GAPDH) primers as probes, and the hybridization method was utilized to achieve SNP genotyping. Using multiplex PCR, and single-labeled fluorescent probes plus universal reverse primer-PCR, we prepared DNA templates for hybridization, and adopted an overnight hybridization method (Fig. S7, Table S8 and Table S10). The results showed that the positive reference DNA of the three genotypes could obtain specific and accurate hybridization signals. The hybridization signals of homozygous wild-type, heterozygous, and 
Table 2 Call Rate and Accuracy of UPIP-qPCR, TaqMan Probe-qPCR and KASP

\begin{tabular}{|c|c|c|c|c|c|c|}
\hline \multirow[t]{2}{*}{ SNPs } & \multicolumn{3}{|c|}{ Call rate $(n=224)$} & \multicolumn{3}{|c|}{ Accuracy $(n=224)$} \\
\hline & UPIP-qPCR & TaqMan & KASP & UPIP-qPCR & TaqMan & KASP \\
\hline rs671 & $99.11 \%$ & $98.21 \%$ & $79.46 \%$ & $100.00 \%$ & $100.00 \%$ & $94.94 \%$ \\
\hline rs1057910 & $100.00 \%$ & $98.66 \%$ & $75.45 \%$ & $100.00 \%$ & $100.00 \%$ & $97.63 \%$ \\
\hline rs9923231 & $100.00 \%$ & $97.77 \%$ & $83.48 \%$ & $100.00 \%$ & $100.00 \%$ & $92.51 \%$ \\
\hline rs1801131 & $100.00 \%$ & $97.32 \%$ & $88.39 \%$ & $100.00 \%$ & $100.00 \%$ & $96.46 \%$ \\
\hline rs1801133 & $99.55 \%$ & $95.09 \%$ & $73.66 \%$ & $100.00 \%$ & $100.00 \%$ & $93.94 \%$ \\
\hline rs1801394 & $100.00 \%$ & $96.88 \%$ & $89.73 \%$ & $100.00 \%$ & $100.00 \%$ & $94.53 \%$ \\
\hline
\end{tabular}

homozygous mutant DNA were FAM-positive and HEX-negative (green dots), FAM- and HEX-positive (yellow dots), and FAM-negative and HEX-positive (red dots), respectively. (Fig. 4b). There were no signals on the blank control microarray that used water as a template, except for the FAM- and HEX-positive control dots (Fig. 4b). FAM (green), HEX (red), FAM and HEX (yellow) and no signal (black background) were displayed at the positions of FAM-positive, HEX-positive, GAPDH and negative reference probes fixed on each microarray, and were consistent with the expected results (Fig. 4b). The hybridization signals of the two types of human genomic DNA (No. 1 and No. 2) were also specific, and consistent with the Sanger sequencing results (Fig. 4b, Table S11, and Additional file 3). Sixteen SNPs can be genotyped for one DNA sample by one microarray simultaneously using this method, thus increasing the detection throughput.

Please see "Additional file 2 "to get more information about results of this study.

\section{Discussion}

In the UPIP-qPCR, intermediate primers were designed to ensure the accuracy of the signal. This was because these primers would only combine with the template DNA to generate specific signals, when the products of the first stage were of the correct DNA segments. In principle, the closer the 3 '-end of the intermediate primer to the SNP locus, the higher the amplification efficiency; therefore, we suggest that the intermediate primers should be designed $\leq 30$ bases from their 3 '-end to the SNP loci (Fig. S3 and Table S4).

Although two-stage reactions were required, the sensitivity of UPIP-qPCR reached three copies per $10 \mu \mathrm{L}$ reaction system, which could compensate this operational defect. Call rates were close to $100 \%$, but this high figure was dependent on the quality of the DNA used in the assay; it should be of high purity. Indeed, the call rate and accuracy of UPIP-qPCR were higher than those of TaqMan probe-qPCR (the standard assay presently in use) and KASP. Other cost-effective, genotyping tools proved to be less accurate and to have lower call rates than TaqMan, such as high-resolution melting (HRM) technology. For this reason, these assays were not directly included in this study [23, 24]. NGS technology is characterized by high-throughput sequencing, and can detect millions of DNA sequences simultaneously. It has the advantages of massive unknow-gene mutation screening and massive SNP genotyping. However, NGS technology is not suitable for and seldom used in genotyping small numbers of known SNPs, owing the long reaction period, high cost of reagents and expensive machines $[25,26]$. There is no comparability between UPIP-qPCR and NGS, because they have specific application fields in SNP genotyping, the former for seldom known SNPs, and the latter for massive unknowns.

The first stage of UPIP-qPCR can be performed on a single target or multiplexed depending on the requirements of the clinician. A single PCR can be adopted when the number of SNPs detected is low and the amount of DNA available is sufficient; otherwis, multiplex PCR can be adopted when the number of SNPs detected is high, and the quantity of DNA available is limited.

The microarray hybridization experiment with intermediate primers as probes provided a basis for developing a new SNP screening method with higher throughput and high accuracy, which would further reduce the price of SNP genotyping. In addition, intermediate primers can also be fixed in multi-hole fluorescence quantitative microfluidic reaction plates, such as Thermofisher QuantStudio $12 \mathrm{~K}$. In this case, only the universal primer, universal probes and stage I multiplex PCR products need to be added to the reaction system, so that in addition to high-throughput SNP genotyping, high-throughput detection of copy number variation $(\mathrm{CNV})$ can also be carried out, thus extending the application range of UPIP-qPCR. Although, we are looking forward to developing a high-throughput realtime planar fluorescent qPCR and hybridizationsequencing technology based on intermediate primers, achieving accurate results and analysis would be a challenging task. 


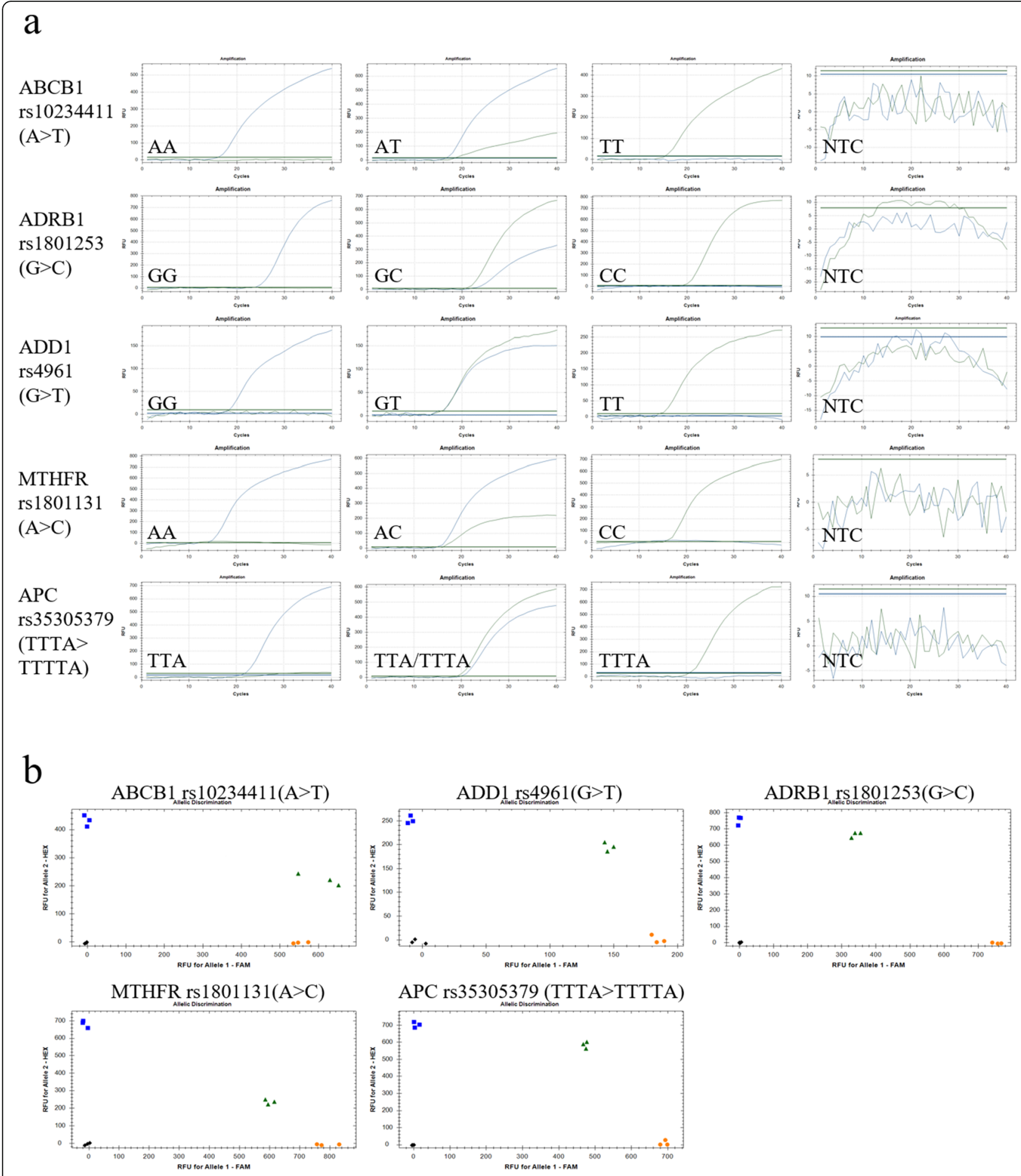

Fig. 3 UPIP-qPCR possessed wide applicability in identifying all kinds of variations in SNPs. UPIP-qPCR presented specific amplification signals and typical S-type curves in the genotype detection of 5 different SNPs with all kinds of variations except G-A and C-T, including interchanges between A-C, A-T, G-C, G-T, and base InDels mutation (a). All three different genotypes of these SNPs were clustered in the scatter diagrams, and the results of genotyping were correct compared to Sanger sequencing(b). In each scatter diagram, WT-, heterogeneous-, and mutant-genotypes were represented by yellow dots, green triangles and blue squares, respectively. Each sample was detected with three duplicates at one experiment, and the experiments were repeated more than three times 


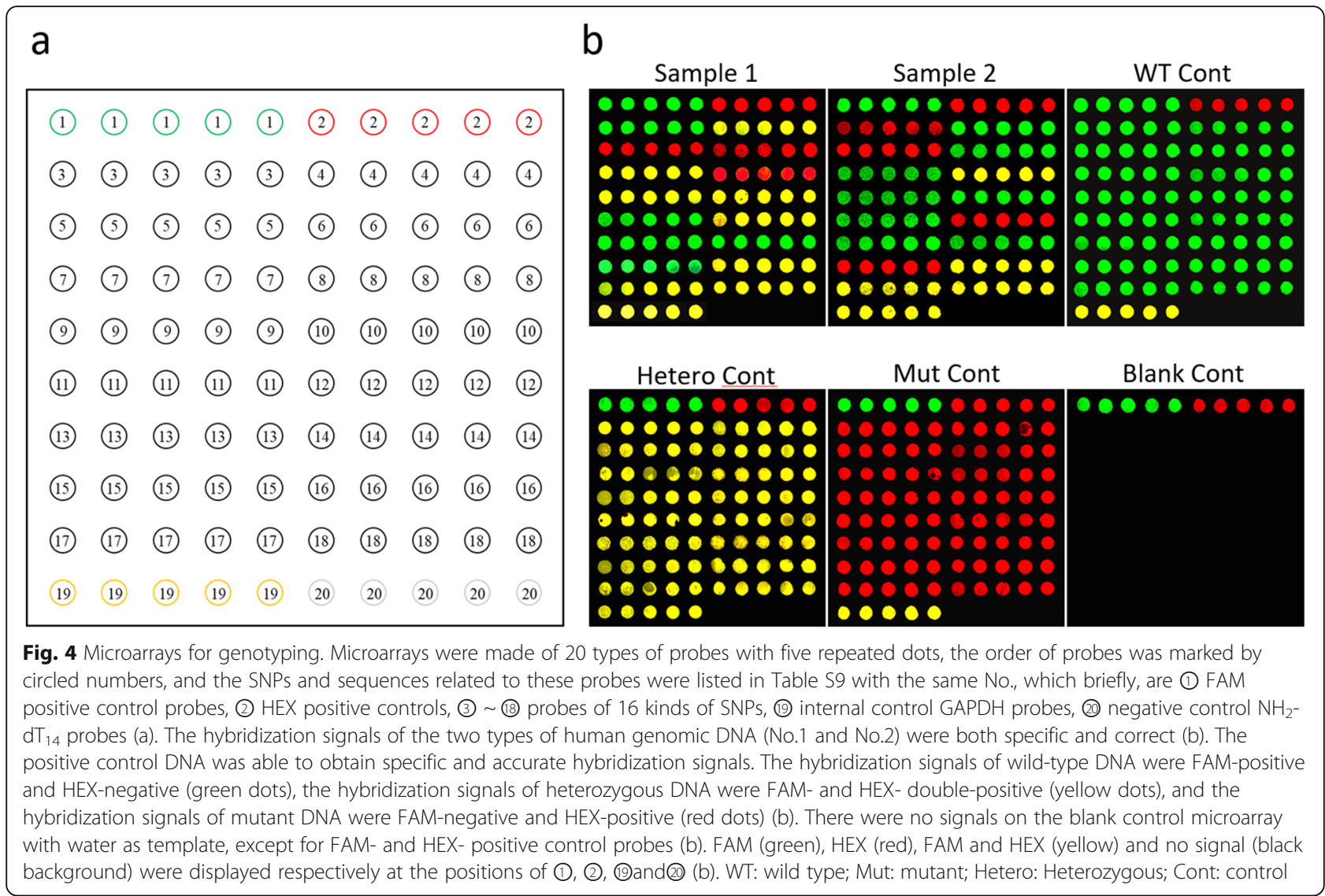

\section{Conclusions}

In this study, we successfully developed an efficient and cost-effective method for SNP genotyping, called universal probe-based intermediate primer-triggered qPCR (UPIP-qPCR). The sensitivity of UPIP-qPCR in SNP genotyping was $0.01 \mathrm{ng}$, the call rate was more than $99.1 \%$, and the accuracy was more than $99.9 \%$, which were both higher than those of TaqMan probe-qPCR. UPIP-qPCR can recognize all type of SNP variants (interchanges between A-G, A-C, A-T, G-C, G-T or C-T) and InDels. Microarrays based on intermediate primers were feasible for SNP genotyping, which may promote a novel high-throughput SNP genotyping method in the future.

In summary, the UPIP-qPCR developed in this study is a novel SNP genotyping technology with low cost, fast detection, and high accuracy, which will help to reduce the cost of clinical detection, reduce the burden of patients, and promote the development of precision medicine.

\section{Methods}

\section{PCR and Sanger sequencing}

The nucleotide sequences containing specific SNPs were acquired from the dbSNP database of the National
Center for Biotechnology Information (NCBI, Bethesda, MD, USA) (https://www.ncbi.nlm.nih.gov/snp/). The primers flanking the SNP site (Fla-primers) and sequencing primers (Seq-primers) were designed using Primer Premier version 5 software (Table S1). Polymerase chain reactions (PCR) were performed to amplify 151-921 bp products containing the targeted SNPs. The nucleotide sequences of the PCR products containing specific SNPs were obtained using a 3730xl DNA analyzer (Applied Biosystems). All blood samples were collected at the Affiliated Yantai Yuhuangding Hospital of Qingdao University. Genomic DNA was isolated from peripheral whole blood samples using the Gentra Puregene Blood Kit (Qiagen Corp, CA) according to the manufacturer's instructions.

\section{UPIP-qPCR}

The UPIP-qPCR consisted of two separate reactions. The first stage (stage I) of UPIP-qPCR was a general PCR. The reaction conditions were as follows: initial denaturation at $95^{\circ} \mathrm{C}$ for $3 \mathrm{~min}$, followed by $10-22$ cycles of denaturation at $98^{\circ} \mathrm{C}$ for $5 \mathrm{~s}$, annealing at $67^{\circ} \mathrm{C}$ for $25 \mathrm{~s}$, elongation at $72^{\circ} \mathrm{C}$ for $20 \mathrm{~s}$, and final elongation at $72{ }^{\circ} \mathrm{C}$ for $1 \mathrm{~min}$. PCR products were diluted ten times 
with $\mathrm{ddH}_{2} \mathrm{O}$ and used as templates in the second-stage reaction.

The second stage (stage II) of UPIP-qPCR was performed using a qPCR. The reaction conditions were as follows: initial denaturation at $95^{\circ} \mathrm{C}$ for $3 \mathrm{~min}$, followed by $35-40$ cycles of denaturation at $98^{\circ} \mathrm{C}$ for $5 \mathrm{~s}$, annealing at $49^{\circ} \mathrm{C}$ for $25 \mathrm{~s}$, elongation at $72^{\circ} \mathrm{C}$ for $1 \mathrm{~s}$ and fluorescent signals were obtained via plate reading. Sequences of primers used for UPIP-qPCR are listed in Table S2. Sequences of FAM- and HEX-labeled universal probes and universal primers are listed in Table S3.

\section{Feasibility verification of UPIP-qPCR}

For this part, we designed the Sanger sequencing related primers (Table S1) for rs671 and rs2031920, through Sanger sequencing to obtain homozygous wild-, heterozygous, and homozygous mutant- types of human genome DNA positive standards. A pair of upstream site-specific primers were designed (Table S2). The first stage of the reaction was carried out on an ordinary PCR instrument, with a total of 10 cycles, at an annealing temperature of $67^{\circ} \mathrm{C}$, to complete the initial amplification of DNA fragments. In the second stage, the signals were collected in real-time as the reaction was carried out on a qPCR instrument with 40 cycles, at an annealing temperature of $49^{\circ} \mathrm{C}$.

\section{Sensitivity analysis}

The genomic DNA samples of the three genotypes of ALDH2 rs671 were used for UPIP-qPCR sensitivity analysis, and $\mathrm{ddH}_{2} \mathrm{O}$ was used for NTC. In the first stage of UPIP-qPCR, the concentration gradients of genomic DNA were $100 \mathrm{ng}, 10 \mathrm{ng}, 1 \mathrm{ng}, 0.1 \mathrm{ng}, 0.01 \mathrm{ng}, 0.003 \mathrm{ng}$ and $0.001 \mathrm{ng}$ per $10 \mu \mathrm{L}$ reaction system. TaqMan probeqPCR was set as control method for the sensitivity analysis. The accuracies mentioned in this section indicates the reproducibility of results when the assay is repeated using the same DNA concentration. Sequences of primers and probes for TaqMan probe-qPCR are listed in Table S5.

\section{Analysis of call rate and accuracy for UPIP-qPCR}

UPIP-qPCR was used to detect the genotypes of $224 \mathrm{hu}-$ man genomic DNA samples at the rs671, rs1057910, rs9923231, rs1801131, rs1801133, and rs1801394 loci. The reaction system and detailed thermal cycle parameters can be found in "UPIP-qPCR". The number of effective results with the total number of samples were compared to obtain the call rate of the UPIP-qPCR method, and the genotyping results were compared with the Sanger sequencing results of the same samples to obtain the accuracy rate of the UPIP-qPCR method. TaqMan probe-qPCR and KASP were used as control methods for the analysis of the call rate and accuracy.
Sequences of primers and probes for TaqMan probeqPCR and KASP are listed in Table S5 and S6, respectively.

\section{Wide applicability analysis of UPIP-qPCR in SNP genotyping}

Based on UPIP-qPCR, we designed primers (Table S2) and genotyped 13 different SNPs. These SNPs covered all SNP mutation types. The system volumes of the first and second-stage UPIP-qPCR reactions were all $10 \mu \mathrm{L}$, and the cycle numbers of the first and second-stage reactions were 10 and 40, respectively. Please refer to "UPIP-qPCR" for the reagent composition of the system.

\section{SNP microarray assay based on intermediate primers}

The microarrays integrated 20 types of probes with five duplicates, including these probes included intermediate primers of 16 types of SNPs, complementary sequences of FAM and HEX single-labeled primers as a positive reference, intermediate primers of GAPDH as an internal reference, and amino modified 14-poly deoxythymine $\left(\mathrm{NH}_{2}-\mathrm{dT}_{14}\right)$ as negative control probes. See Table S9 for the sequences of the probes.

The preparation process of the PCR products for hybridization was divided into three stages. The first stage was multiplex PCR. In the first stage, normal human genome DNA (No. 1 and No. 2) was used as the template DNA of the experimental group, and the template DNA of the control groups was divided into three types, namely, the mixture of wild-type, heterozygous and mutant positive control DNA of 16 SNP sites, and the wild type positive control DNA of GAPDH. $d_{d d H_{2}} \mathrm{O}$ was used as the blank control template. The second stage was a product treatment process, that is, using exonuclease I to digest the products of the first stage which would be used as templates in the third stage reactions. The third-stage was the fluorescence labeling PCR reaction. In the third stage, FAM and HEX singlelabeled primers and universal reverse primer (Table S3) were combined to amplify the templates to obtain sufficient DNA fragments for microarray hybridization.

Products of the third stage were denatured at $95^{\circ} \mathrm{C}$ for $5 \mathrm{~min}$, cooled on ice for $2 \mathrm{~min}$, and then mixed with an equal volume hybridizing buffer to form a hybridizing solution. Hybridizing solution $(20 \mu \mathrm{L})$ was aliquoted into the microarrays, covered with coverslips, and incubated for 16-20 h. Images of the FAM and HEX signals were captured using a confocal microscope. Dots with only FAM signals (green) denoted the homozygous wild type, those with only HEX signals (red) were homozygous mutants, and those with both signals (yellow) were heterozygous. 


\section{Data analyses}

One-way ANOVA tests were used for data comparation between the groups. Differences were considered statistically significant when $P<0.05$. Data analyses were performed using SPSS 16.0.

Please see "Additional file 1 "to get more detailed methods.

\section{Abbreviations}

UPIP-qPCR: Universal probe-based intermediate primer-triggered GPCR SNP: Single nucleotide polymorphism; ARMS: Amplification-refractory mutation system; KASP: Kompetitive allele-specific PCR; RFU: Relative fluorescence units; NTC: No-template control; HRM: High-resolution melting; NGS: Next-generation sequencing; CNV: Copy number variation

\section{Supplementary Information}

The online version contains supplementary material available at https://doi. org/10.1186/s12864-021-08148-2.

Additional file 1. Detailed Materials and Methods for UPIP-qPCR.

Additional file 2. Additional results of UPIP-qPCR.

Additional file 3. GenBank accession numbers.

Additional file 4. Supplementary figure legends.

Additional file 5. Supplementary Tables.

\section{Acknowledgments}

We want to acknowledge Dr. Haoxun Li (Beijing Jiacheng Biotechnology Co., Ltd) for providing the information about KASP.

\section{Authors' contributions}

$\mathrm{BL}$ designed and completed most of the experiments. $Y \mathrm{~L}, \mathrm{XH}, \mathrm{WW}$ and $\mathrm{YL}$ prepared human genomic DNA samples and performed partial GPCR experiments. BL wrote the manuscript. JD, LC, HL and JW revised the manuscript. $B L, Y W$, and $P L$ revised the manuscript and provided financial support. All authors have read and approved the manuscript.

\section{Funding}

This work was funded by the National Natural Science Foundation of China (31430041), the Major Research Program of the National Natural Science Foundation of China (91849209) and the China Postdoctoral Science Foundation (2018 M632613). The funding bodies played no role in the design of the study and collection, analysis, and interpretation of data and in writing the manuscript.

\section{Availability of data and materials}

The datasets supporting the conclusions of this article are available in the NCBI GenBank repository with accession number OK351361 - OK352256, OK480620 - OK480877, OK492234 - OK492457, https://www.ncbi.nlm.nih.gov/ nuccore/. Definitions for these accession numbers can be found in Additional file 3 .

\section{Declarations}

\section{Ethics approval and consent to participate}

This study was approved by the Ethics Committee of the Affiliated Yantai Yuhuangding Hospital of Qingdao University (Yantai, China), and all subjects (224 normal individuals of Chinese Han) provided written informed consent prior to enrollment. This study was conducted following the Declaration of Helsinki.

\section{Consent for publication}

Not Applicable.

\section{Competing interests}

The authors declare that they have no competing or conflicts of interest.

\section{Author details}

'Key Laboratory of Biological Medicines in Universities of Shandong Province, Weifang Key Laboratory of Antibody Medicines, School of Bioscience and Technology, Weifang Medical University, Jinan 261053, Shandong, China. ${ }^{2}$ Institute for Translational Medicine, Qingdao University, Qingdao 266021, China. ${ }^{3}$ School of Basic Medicine, Qingdao University, Qingdao 266021,

China. ${ }^{4}$ College of Food Science and Engineering, Qingdao Agricultural University, Qingdao 266109, China.

Received: 16 December 2020 Accepted: 3 November 2021

Published online: 24 November 2021

\section{References}

1. Scheen AJ. Precision medicine: the future in diabetes care? Diabetes Res Clin Pract. 2016;117:12-21. https://doi.org/10.1016/j.diabres.2016.04.033.

2. Ho DSW, Schierding W, Wake M, Saffery R, O'Sullivan J. Machine learning SNP based prediction for precision medicine. Front Genet. 2019;10:267. https://doi.org/10.3389/fgene.2019.00267.

3. Tanner JA, Tyndale RF. Variation in CYP2A6 Activity and Personalized Medicine. J Personalized Med. 2017;7(4):7(4). https://doi.org/10.3390/jpm704 0018 .

4. Li B, Wang X, Hao X, Liu Y, Wang Y, Shan C, et al. A novel C.2179T>C mutation blocked the intracellular transport of PHEX protein and caused $X$ linked hypophosphatemic rickets in a Chinese family. Mol Genet Genomic Med. 2020;n/a(n/a):e1262.

5. Perkel J. SNP genotyping: six technologies that keyed a revolution. Nat Methods. 2008;5(5):447-53. https://doi.org/10.1038/nmeth0508-447.

6. Matsuda K. PCR-based detection methods for single-nucleotide polymorphism or mutation: real-time PCR and its substantial contribution toward technological refinement. Adv Clin Chem. 2017;80:45-72. https://doi. org/10.1016/bs.acc.2016.11.002.

7. You Q, Yang X, Peng Z, Xu L, Wang J. Development and applications of a high throughput genotyping tool for Polyploid crops: single nucleotide polymorphism (SNP) Array. Front Plant Sci. 2018;9:104. https://doi.org/10.33 89/fpls.2018.00104.

8. Kim S, Misra A. SNP genotyping: technologies and biomedical applications. Annu Rev Biomed Eng. 2007:9(1):289-320. https://doi.org/10.1146/annurev. bioeng.9.060906.152037.

9. Sanger F, Nicklen S, Coulson AR. DNA sequencing with chain-terminating inhibitors. Proc Natl Acad Sci U S A. 1977;74(12):5463-7. https://doi.org/10.1 073/pnas.74.12.5463.

10. Wu H, Wu X, Liang Z. Impact of germline and somatic BRCA1/2 mutations: tumor spectrum and detection platforms. Gene Ther. 2017;24(10):601-9. https://doi.org/10.1038/gt.2017.73.

11. Lee $L G$, Connell CR, Bloch W. Allelic discrimination by nick-translation PCR with fluorogenic probes. Nucleic Acids Res. 1993;21(16):3761-6. https://doi. org/10.1093/nar/21.16.3761.

12. Dooms $M$, Chango A. Abdel-nour a: [quantitative $P C R(q P C R$ ) and the guide to good practices MIQE: adapting and relevance in the clinical biology context]. Ann Biol Clin. 2014;72(3):265-9. https://doi.org/10.1684/abc.2014. 0955.

13. Chu MKM, Wong EYL, Wong SCC, Alikian M, Gale RP, Apperley JF, et al. Molecular techniques for the personalised management of patients with chronic myeloid leukaemia. Expert Rev Mol Diagn. 2017;11:4-20. https://doi. org/10.1016/j.bdq.2017.01.001.

14. Sanjuan-Jimenez R, Colmenero JD, Morata P. Lessons learned with molecular methods targeting the BCSP-31 membrane protein for diagnosis of human brucellosis. Clin Chimica Acta Int J Clin Chem. 2017;469:1-9.

15. Zhang L, Cui G, Li Z, Wang H, Ding H, Wang DW. Comparison of highresolution melting analysis, TaqMan allelic discrimination assay, and sanger sequencing for Clopidogrel efficacy genotyping in routine molecular diagnostics. J Mol Diagnostics JMD. 2013;15(5):600-6. https://doi.org/10.101 6/j.jmoldx.2013.04.005.

16. Newton CR, Graham A, Heptinstall LE, Powell SJ, Summers C, Kalsheker N, et al. Analysis of any point mutation in DNA. The amplification refractory mutation system (ARMS). Nucleic Acids Res. 1989;17(7):2503-16. https://doi. org/10.1093/nar/17.7.2503.

17. Newton CR, Heptinstall LE, Summers C, Super M, Schwarz M, Anwar R, et al. Amplification refractory mutation system for prenatal diagnosis and carrier assessment in cystic fibrosis. Lancet. 1989:2(8678-8679):1481-3. https://doi. org/10.1016/s0140-6736(89)92931-0. 
18. Ferrie RM, Schwarz MJ, Robertson NH, Vaudin S, Super M, Malone G, et al. Development, multiplexing, and application of ARMS tests for common mutations in the CFTR gene. Am J Hum Genet. 1992;51 (2):251-62.

19. Zhang Y, Zhang D, Li W, Chen J, Peng Y, Cao W. A novel real-time quantitative PCR method using attached universal template probe. Nucleic Acids Res. 2003;31(20):123e-123. https://doi.org/10.1093/nar/gng123.

20. He C, Holme J, Anthony J. SNP genotyping: the KASP assay. Methods Mol Biol (Clifton, NJ). 2014;1145:75-86.

21. Ertiro BT, Ogugo V, Worku M, Das B, Olsen M, Labuschagne M, et al. Comparison of Kompetitive allele specific PCR (KASP) and genotyping by sequencing (GBS) for quality control analysis in maize. BMC Genomics. 2015; 16(1):908. https://doi.org/10.1186/s12864-015-2180-2.

22. Ragoussis J. Genotyping technologies for all. Drug Discov Today Technol. 2006;3(2):115-22. https://doi.org/10.1016/j.ddtec.2006.06.013.

23. Gundry CN, Vandersteen JG, Reed GH, Pryor RJ, Chen J, Wittwer CT. Amplicon melting analysis with labeled primers: a closed-tube method for differentiating homozygotes and heterozygotes. Clin Chem. 2003;49(3):396406. https://doi.org/10.1373/49.3.396.

24. Martino A, Mancuso T, Rossi AM. Application of high-resolution melting to large-scale, high-throughput SNP genotyping: a comparison with the TaqMan method. J Biomol Screen. 2010;15(6):623-9. https://doi.org/10.11 77/1087057110365900.

25. Cirulli ET, Lasseigne BN, Petrovski S, Sapp PC, Dion PA, Leblond CS, et al. Exome sequencing in amyotrophic lateral sclerosis identifies risk genes and pathways. Science. 2015;347(6229):1436-41. https://doi.org/10.1126/ science.aaa3650.

26. Perkel JM. Single-cell sequencing made simple. Nature. 2017;547(7661):1256. https://doi.org/10.1038/547125a.

\section{Publisher's Note}

Springer Nature remains neutral with regard to jurisdictional claims in published maps and institutional affiliations.

Ready to submit your research? Choose BMC and benefit from:

- fast, convenient online submission

- thorough peer review by experienced researchers in your field

- rapid publication on acceptance

- support for research data, including large and complex data types

- gold Open Access which fosters wider collaboration and increased citations

- maximum visibility for your research: over $100 \mathrm{M}$ website views per year

At $\mathrm{BMC}$, research is always in progress.

Learn more biomedcentral.com/submissions 